\title{
Classification of the liver tumors Using Co-Occurrence Matrices of textural Microstructures
}

\author{
Delia Mitrea, Sergiu Nedevschi and Mihail Abrudean \\ Faculty of Automation and Computer Science, Technical University of Cluj-Napoca, Cluj-Napoca 400027, Romania
}

\begin{abstract}
Cancer detection is a major issue in the medical research nowadays, as cancer is a severe disease, leading to death in many situations. In order to perform tumor recognition based on ultrasound images we defined the textural model of the malignant tumors consisting of the relevant textural features able to best separate these tumors from visually similar tissues and of their specific values: mean, standard deviation, probability distribution. In this paper, we assessed the role that various types of textural microstructure co-occurrence matrices have in the improvement of the textural model of the liver tumors. We considered the classes of HCC (hepato cellular carcinoma), of the cirrhotic parenchyma on which HCC had evolved and of hemangioma, a benign liver tumor. The classifiers of Multilayer Perceptron, Support Vector Machines, the decision trees based algorithms Random Forest and C4.5, respectively adaboost in combination with $\mathrm{C} 4.5$, were used for classification performance assessment.
\end{abstract}

Key words: Texture, textural microstructure co-occurrence matrices, liver tumors, ultrasound images, classification performance.

\section{Introduction}

Cancer is a frequent disease, well known for its severity. The HCC is the most important malignant liver tumor, appearing in $75 \%$ of the liver cancer cases. The most reliable method for liver cancer detection is the biopsy, but this method is invasive and dangerous. We developed computerized methods, based on ultrasound images, for non-invasive tumor detection. The ultrasonography is a reliable technique for patient examination, being also easy to apply, not expensive and having the possibility of repeatability. Other examination methods based on medical imaging, such as CT (computer tomography), or MRI (magnetic resonance imaging) can be irradiating or expensive [1]. The texture is an important feature of the internal organ tissues, providing subtle information about the pathological state. In our research, we defined the textural model of some important affections [2, 3] consisting of the relevant textural features, able to best characterize these diseases and of their specific values:

Corresponding author: Delia Mitrea, Ph.D., senior lecturer, research fields: image processing and pattern recognition, human-computer interaction. E-mail: Delia.Mitrea@cs.utcluj.ro. mean, standard deviation, probability distribution. We computed the classical textural features $[4,5]$ and we also elaborated new methods for texture characterization, such as the GLCM (grey level cooccurrence matrix) of superior order, respectively the EOCM (edge orientation cooccurrence matrix) of order two and three $[2,3]$. In the current work, we defined cooccurrence matrices of the textural microstructures, computed after applying the Laws convolution filters [6], followed by an improved version of the k-means clustering algorithm [7, 8]. Because the frequency of the spot and ripple microstructures provided the best results in our former experiments, we employed them in our newly defined second order statistics. For assessing the resulted classification performance, we implemented powerful classifiers, such as: the MLP (multilayer perceptron), the SVM (support vector machines), the RF (random forest) and the C4.5 algorithms for decision trees, as well as the adaboost metaclassifier in combination with C4.5. The pathology classes taken into consideration for the validation were: the HCC; the cirrhotic parenchyma on which HCC evolved; the 

$S_{5} R_{5}, R_{5} S_{5}$ [6]. We determined the TMCM matrices considering each of these filters individually (simple TMCM matrices) and also when considering, for each pixel, the feature vector composed by the results obtained after applying all the above mentioned filters, yielding more complex microstructures (complex TMCM matrix CTMCM). We determined the cooccurrence matrices of order two and three. For the TMCM of order two, the following directions were considered: $0^{\circ}, 90^{\circ}, 180^{\circ}, 270^{\circ}$. For the TMCM of order three, the current pixel was considered in the central position. This pixel, together with the other two pixels, were either collinear, or formed a right angle triangle (with the current pixel being the vertex corresponding to the right angle). Thus, the considered orientations for the displacement vectors were: $\left(0^{\circ}\right.$, $\left.180^{\circ}\right),\left(90^{\circ}, 270^{\circ}\right),\left(45^{\circ}, 225^{\circ}\right),\left(135^{\circ}, 315^{\circ}\right)$ for the case of collinear pixels; $\left(0^{\circ}, 90^{\circ}\right),\left(90^{\circ}, 180^{\circ}\right),\left(180^{\circ}\right.$, $\left.270^{\circ}\right),\left(0^{\circ}, 270^{\circ}\right),\left(45^{\circ}, 135^{\circ}\right),\left(135^{\circ}, 225^{\circ}\right),\left(225^{\circ}\right.$, $\left.315^{\circ}\right),\left(45^{\circ}, 315^{\circ}\right)$, for the second case. The absolute value of the displacement vectors was considered as being 2, in both cases. The TMCM matrices corresponding to all the considered direction combinations were determined, the final features resulting as an average between the Haralick features of the individual matrices. Besides the new textural features, we considered previously defined textural features, as well as classical textural features, such as: the GLCM of order two and three, the EOCM of order two and three, the edge orientation variability, the edge frequency and contrast, the autocorrelation index, the Hurst fractal index, the frequency of the textural microstructures, the entropy computed after applying the Wavelet transform, at two levels of resolution [5].

\subsection{Relevant feature selection}

For the selection of the relevant textural features, we considered specific methods, which provided the best results in our experiments: Consistency based Feature Subset Evaluation (Consistency) [18], combined with genetic search [19], CFS (correlation based feature selection) [18] combined with genetic search, respectively Gain Ratio Attribute Evaluation [18], combined with the Ranker method [19], in order to assess the individual features by assigning them an information gain [18]. When applying simoultaneously multiple methods, the final relevance score was obtained as an average between the individual scores.

\subsection{Classification performance assessment}

In order to assess the role of the TMCM features concerning the automatic diagnosis of the liver tumors, we used the classifiers of MLP [20], of SVM [20], respectively the decision trees algorithms $\mathrm{RF}$ and $\mathrm{C} 4.5$ [20]. Classifier combination schemes were employed, as well, for increasing the performance of the previously mentioned classifiers. Thus, the adaboost meta-classifier, in combination with the $\mathrm{C} 4.5$ algorithm was also employed, being known for its performance. For classification performance assessment, we used the following parameters: the recognition rate (classification accuracy), the sensitivity (TP rate), the specificity (TN rate), the AUC (area under ROC) [20]. For performance evaluation, the strategy of cross-validation was implemented.

\section{Experiments and Discussions}

For the experiments, we used 250 cases of HCC, together with the cirrhotic parenchyma on which HCC had evolved, respectively 100 cases of hemangioma. All these patients underwent biopsy, for diagnostic confirmation. For each patient, 3 images were considered, for various orientations of the transducer. The images were acquired using a Logiq 7 ultrasound machine, at the frequency of $5.5 \mathrm{MHz}$, the gain of 78 and the depth of $16 \mathrm{~cm}$. On each image, a region of interest, having 50x50 pixels in size, was selected inside the corresponding class of tissue. The textural features were computed independently on orientation, illumination and region of interest size, using our Visual C++ software modules. The feature selection 



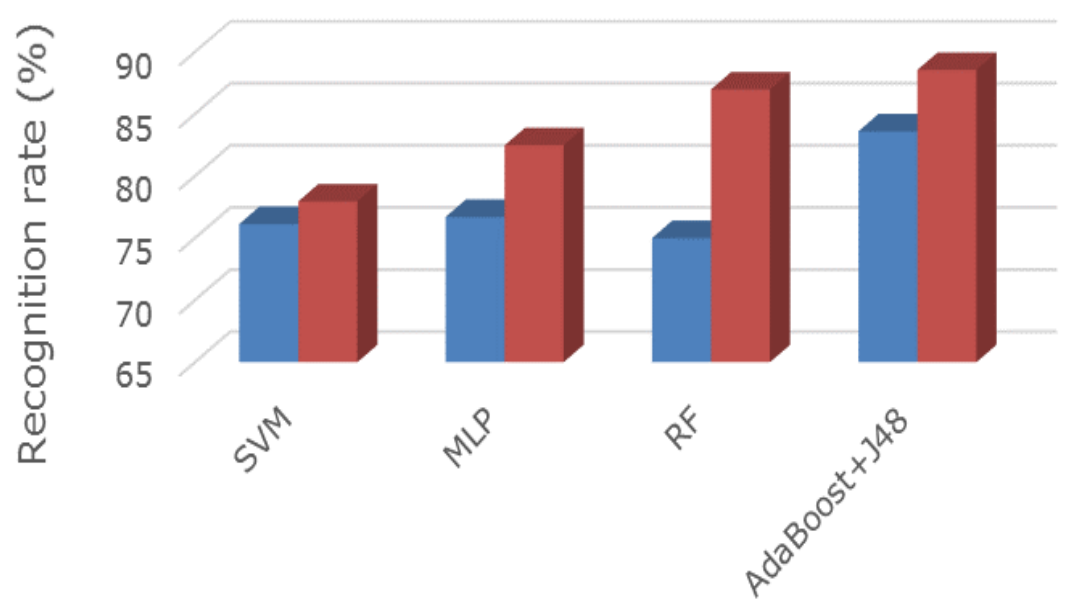

m Old textural features $\square$ Old textural features + new textural features

Fig. 1 The increase in the classification, when comparing HCC and cirrhotic parenchyma, for the simple TMCM.

the second and third order TMCM matrix, based on combined textural microstructures of type S5R5, resulted as being relevant. In this case, the maximum recognition rate, of $96.36 \%$, as well as the maximum sensitivity of $96.4 \%$, were obtained in the case of the SVM classifier, the maximum specificity, of $98.2 \%$, resulted in the case of the RF classifier and the maximum AUC, of $99.4 \%$, resulted in the case of the MLP classifier. In all these cases, the accuracy due to the combination between the old and newly defined textural features was better than the accuracy due to the old textural features (the average recognition rate was $95.22 \%$ in the first situation and $85.66 \%$ in the second situation).

\subsection{Experiments involving the CTMCM matrix}

Comparison between $\mathrm{HCC}$ and the cirrhotic parenchyma on which HCC had evolved

The set of the most relevant textural features for the differentiation between HCC and cirrhotic parenchyma, obtained in the case when using the CTMCM parameters, was derived using the CFS method combined with genetic search, as well as the Gain Ratio Attribute Evaluation technique combined with Ranker[14]. We remarked the presence of the energy and contrast parameters derived from the CTMCM matrix. The CTMCM energy, which was lower in the case of $\mathrm{HCC}$, denoted the heterogeneity of the tumor tissue, while the CTMCM contrast, which was higher in the case of HCC, denoted the complex structure of this tumor. In this case, the maximum values of the classification performance parameters (recognition rate: $83.95 \%$, sensitivity: $88.5 \%$, specificity: $79.4 \%$, AUC: $90.4 \%$ ) were obtained in the case of the AdaBoost metaclassifier combined with decison trees (J48). We also assessed the increase in accuracy due to the new textural features, in comparison with that provided only by the old textural feature set. The classification performance was improved in most of the cases, in comparison with the case when only the old textural features were employed: obvious improvements were noticed for the RF classifier and for AdaBoost combined with decision trees, a slight accuracy improvement was remarked for the MLP classifier (Fig. 2). Also, the classification performance was better than that illustrated in Ref. [17].

Comparison between HCC and hemangioma

The maximum recognition rate, of $88.41 \%$, was obtained in the case of the AdaBoost classifier combined with decision trees, while the average classification accuracy, for all the considered classifiers, was 95.53\%. Concerning the relevant textural features obtained in this situation, we noticed 

